\title{
Definition of Liability Index for Three-phase Unbalanced Power System
}

\author{
Pengyu Zhang \\ School of Electrical Engineering, North China Electric Power University, Baoding 071003, China \\ gotosea@foxmail.com
}

Keywords: Negative sequence impedance, allocation of responsibility, rewards and punishment mechanism, quantitative index.

\begin{abstract}
The unbalance responsibility of main unbalanced source is an important task of power quality management. This paper presents an unbalanced voltage liability index to measure the imbalance liability problem. Taking into account the power grid can quality monitoring system provides only statistical values of voltage and current of the actual situation, put forward a based on electricity to quality data exchange format, quantitative estimation algorithm, so that the proposed method can use the electric energy quality monitoring system realize daily imbalance, monitoring and determination.
\end{abstract}

\section{Introduction}

The problem of three-phase unbalance in current power network is becoming increasingly serious. Engineering general according to the number of individual users of the monitoring. According to the unbalance calculation method for voltage unbalance degree (GB/IEC/IEEE respectively), this paper proposes five calculated assessment, electricity enterprise according to determine three-phase unbalanced control scheme, pursuant to the power sector of economic rewards and punishments.

However, the public connection point in general there may be a plurality of three-phase unbalanced source user, the three-phase unbalanced load between how to accurately of apportion blame is three-phase unbalanced governance and economic rewards and punishments according to. Therefore, a three phase imbalance of the "incentive and power supply program" has been put on the agenda.

The current for three-phase unbalance problem and hot research focus in the solution design, as by the PWM inverter to the distribution network into the compensation of zero sequence current three phase unbalance current, automatically change the phase technology, SVG without reactive power compensation etc.. And the quantitative study of the responsibility is not much. The concept of unbalanced launch carrier is presented in the following ${ }^{[1-2]}$, and the whole effect caused by the imbalance of the system and the imbalance of the load is determined. According to the background, exploiting the analogy imbalance of propagating mechanism and harmonic propagation mechanism, combined with harmonic voltage, index of unbalanced responsibility indicators proposed, follow-up will draw on the harmonic pollution, the quantitative estimation principle to balance the allocation of responsibility for.

\section{Proposed liability index}

The three phase unbalance is caused by the unbalance of the power supply link and the electric link in two aspects. The imbalance of power supply link is caused mainly by the imbalance of power supply line. The unbalance of electric link is caused mainly by the load asymmetry, including electric arc furnace, electrified railway, etc. The system and the user can be equivalent to negative sequence impedance and constant negative sequence current source parallel to the negative sequence equivalent circuit (Norton equivalent circuit), as shown below. 


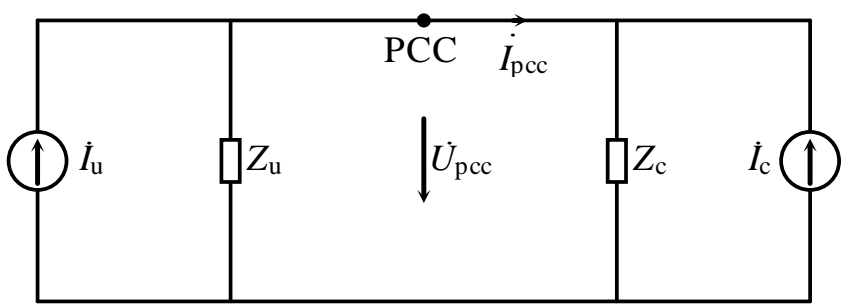

Fig. 1 Norton equivalent circuit
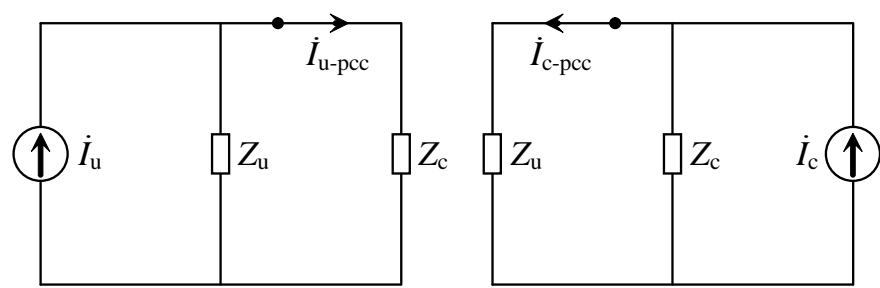

Fig. 2 Determination of unbalance source contribution

In the picture:

$\mathbf{I}_{U}$ and $\mathbf{I}_{C}$ are respectively the equivalent negative sequence current source for the power supply system and the user side;

$Z_{U}$ and $Z_{C}$ are negative sequence impedances for the power supply system and the user's side;

The negative sequence voltage and negative sequence current were measured at PCC (public connection point) by $\mathbf{U}_{\mathrm{PCC}}$ and I PCC respectively;

$\mathrm{U}_{\mathrm{NU}}$ and $\mathrm{I}_{\mathrm{NU}}$ are negative sequence voltage and negative sequence current for the negative sequence current source of the system side;

The negative sequence voltage and negative sequence current of the $U_{N_{C}}$ and $I_{N C}$ for the negative sequence current source of the user side.

The formula of calculation is as follows:

$$
\begin{aligned}
& \dot{U}_{N U}=\frac{Z_{C} Z_{U}}{Z_{C}+Z_{U}} \dot{I}_{U} \\
& \dot{U}_{P C C}=Z_{U}\left(\dot{I}_{U}+\dot{I}_{P C C}\right) \\
& \dot{U}_{N U}=Z_{C}\left(\frac{\dot{U}_{P C C}-Z_{U} \dot{I}_{P C C}}{Z_{C}+Z_{U}}\right)
\end{aligned}
$$

We can finally get (3) by Formula (1) and (2). After the same derivation, we can also get (4):

$$
\dot{U}_{N C}=Z_{U}\left(\frac{\dot{U}_{P C C}+Z_{C} \dot{I}_{P C C}}{Z_{C}+Z_{U}}\right)
$$

Because the majority of the international standards are aimed at the negative sequence components. From the above model, the responsibility of the negative sequence voltage is obtained by the user's side to the imbalance responsibility of the PCC:

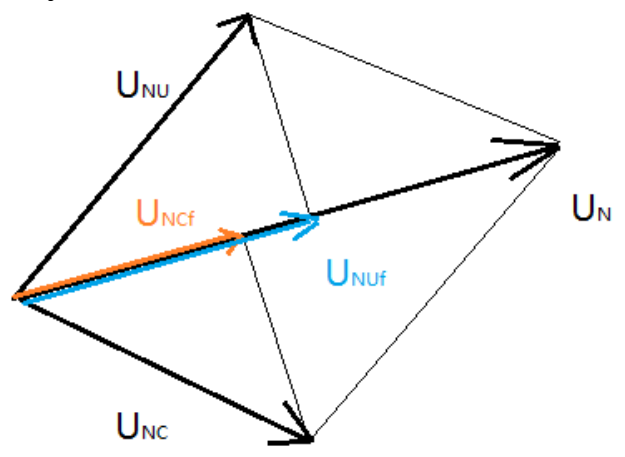

Fig. 3 Define liability index by vector projection 
By using the vector projection method, we can get the responsibility index $\alpha_{c}$.

$$
\alpha_{c}=\frac{U_{N C} \cos \left(\theta_{N C}-\theta_{N}\right)}{U_{N}}
$$

Put the formula (1) to (4) into the formula, can be easily obtained

$$
\alpha_{c}=\frac{Z_{U}\left|\dot{U}_{P C C}+Z_{C} \dot{I}_{P C C}\right| \cos \left(\theta_{C}-\theta_{P C C}\right)}{\left(Z_{C}+Z_{U}\right) U_{P C C}}
$$

So the key to the problem is: 1 ) The negative sequence voltage and current measured at PCC can be solved by FFT and symmetric component. 2)The equivalent negative sequence impedance of the power supply system and the user side. 3)The phase angle.

As the theory is difficult to be supported by the test data in practice, an improved method for solving the above theory is proposed.

\section{Improved algorithm for solving problem}

Based on the discussion of the harmonic impedance ${ }^{[3]}$, we design the iterative algorithm to solve the linear regression of the equivalent negative sequence impedance. The description of the algorithm directly omitted, the conclusion is given.

$\dot{U}_{2 A}$ represents the negative sequence voltage of the A phase is measured at the bus bar at the bus bar, at the bus bar of the common connecting point PCC. $\dot{I}_{2 A}$ represents the fundamental negative sequence current of the A phase at the source of the three-phase unbalance source. $\dot{U}_{2,0}(l)$ is negative sequence background voltage.

Finally, the negative sequence impedance is obtained and the negative sequence background voltage is calculated:

$$
\begin{aligned}
& Z_{2 A}=\frac{1}{c} \sum_{q=1}^{c} Z_{2 A}\left(s_{q}\right) \\
& \dot{U}_{2,0}(k)=\dot{U}_{2 A}(k)-Z_{2 A} \dot{I}_{2 A}(k), \quad k=1,2, \ldots, n
\end{aligned}
$$

Then, on the basis of the above research, we can obtain from the user side, PCC public connection point (that is, the bus) is measured to the negative sequence voltage $U_{N}\left(\dot{U}_{2 A}\right)$ and negative sequence background harmonic voltage $\mathrm{U}_{\mathrm{NU}}\left(\dot{U}_{2,0}\right)$.

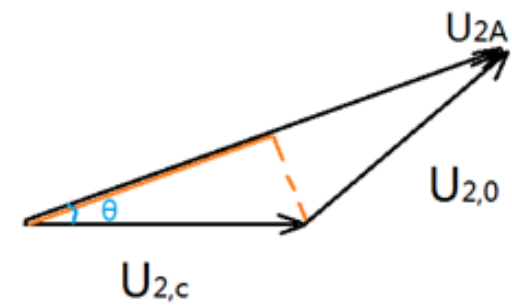

Fig. 4 Definition of liability index by triangle method

Next, estimate the negative sequence voltage RMS of the user contribution $\dot{U}_{2, c}$

$U_{2, c}=\left|Z_{2 A}\right| I_{2 A}$

Can easily get the cosine and the user for the PCC imbalance responsibility:

$$
\begin{aligned}
& \cos \theta=\frac{U_{2 A}^{2}+U_{2, c}^{2}-U_{2,0}^{2}}{2 U_{2 A} U_{2, c}} \\
& \alpha_{c}=\frac{U_{2, c} \cos \theta}{U_{2 A}}
\end{aligned}
$$




\section{Summary}

This article originally proposed a the unbalanced degree of propagating mechanism and harmonic propagation mechanism of analogy, and determine the method of unbalanced responsibility index according to harmonic, voltage index, and quantitative imbalance problem of shared responsibility.

\section{References}

[1] Paranavithana P, Perera S. Location of Sources of Voltage Unbalance in anInterconnected Network[C]//IEEE Power \& Energy Society General Meeting,2009

[2] ZHU Mingxing, QIAN Chenchen, HU Tingfang. Location of sources of unbalance in three phase power networks[J]. Electrotechnical Application, 2012(17):28-31.

[3] Jia Xiufang, Hua Huichun,Cao Dongsheng,et al.Determining harmonic contributions based on complex least squares method[J].Proceedings of the CSEE,2013,33(4):149-155. 